\title{
Working across boundaries - development of an outreach support service for patients requiring home parenteral nutrition
}

\author{
C. Shaw ${ }^{1,2}$, A. Myers ${ }^{1}$, J. L. Shaffer ${ }^{1}$ and A. Bradley ${ }^{1}$ \\ ${ }^{1}$ Salford Royal NHS Foundation Trust, Salford, UK and ${ }^{2}$ Bupa Home Healthcare (formerly Clinovia), \\ Bupa Home Healthcare, Roydon Road, Harlow, Essex
}

While many patients are able to independently manage their home parenteral nutrition (HPN), some are unable to do so. This can be due to a variety of reasons including disabilities, disease processes and age. A pioneering joint venture by one of the intestinal failure units (IFU) and a commercial homecare company (CHC) in 1998, resulted in the appointment of a National Liaison Nurse to work in a joint role. Outreach services deliver treatment usually provided in the hospital setting, in the patient's home. In addition, some patients who previously would not have been able to be safely discharged have now been offered personalised care packages.

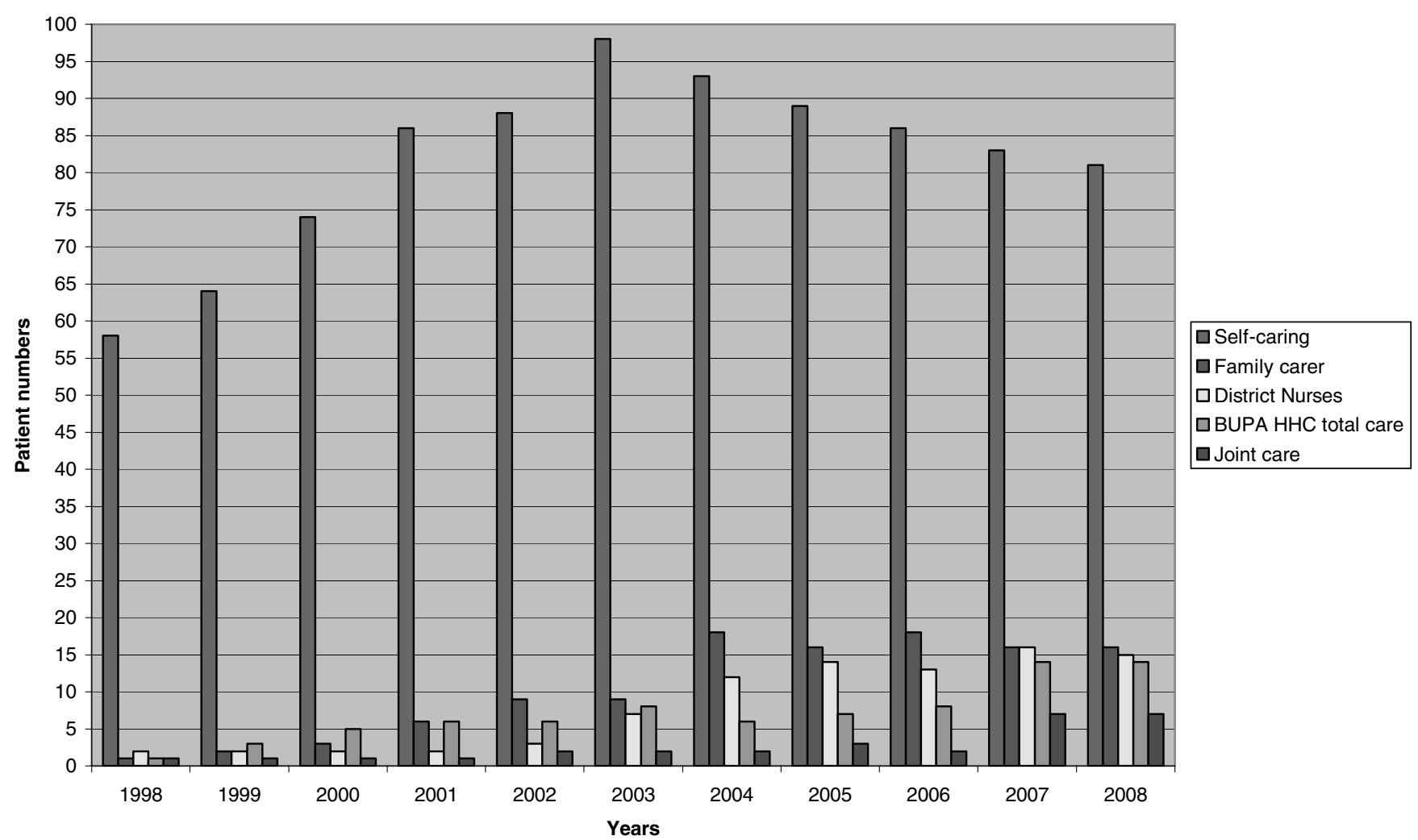

Figure 1 Central line carers

At the start of the venture in 1998, 92\% of 62 patients on HPN were self-caring, by 2008 this had fallen to $61 \%$ of 133 patients (see Figure 1 )

The homecare company nurses have managed complete HPN care packages for 29 patients and also provided aseptic protocol training for district nurses to give part or total care to 49 patients in addition to training for carers of 32 other patients following re-assessment of their changing care needs.

In addition, the following areas have been addressed (1) patient training completed at home, (2) clinic at home' service, (3) blood monitoring, (4) abdominal wound assessment, (5) fluid and electrolyte balance, (6) infusion pump issues, (7) clearing occluded central lines, (8) repairing damaged central lines, (9) diagnosis and management of central line exit site infection, (10) central and peripheral blood cultures for suspected catheter infections. Patients have received the appropriate care in the place most convenient to them, and the need for hospital visits or re-admission to hospital has been reduced. The assurance that the patients receive appropriate support to meet their needs is integral to this role. A total of 281 patients have been supported by this service since 1998

The company has since provided additional nursing resource to further develop the service. The important issue of quality of life in chronic illness has been addressed in this patient group and the needs of an ageing population met. 